Témoigner Témoigner. Entre histoire et mémoire

Getuigen Revue pluridisciplinaire de la Fondation Auschwitz

$123 \mid 2016$

Traduire le témoignage

\title{
Des moments de lucidité
}

Moments of Clarity

\section{Omar Robert Hamilton}

Traducteur : Carine Chauran

\section{(2) OpenEdition}

\section{Journals}

Édition électronique

URL : https://journals.openedition.org/temoigner/5429

DOI : 10.4000/temoigner.5429

ISSN : 2506-6390

Éditeur :

Éditions du Centre d'études et de documentation Mémoire d'Auschwitz, Éditions Kimé

Édition imprimée

Date de publication : 1 octobre 2016

Pagination : 152-157

ISBN : 987 2-9600926-4-6

ISSN : 2031-4183

Référence électronique

Omar Robert Hamilton, «Des moments de lucidité », Témoigner. Entre histoire et mémoire [En ligne],

123 | 2016, mis en ligne le 02 novembre 2021, consulté le 04 novembre 2021. URL : http:// journals.openedition.org/temoigner/5429; DOI : https://doi.org/10.4000/temoigner.5429

Tous droits réservés 
Traduire la révolution

égyptienn
(suite)

\section{Des moments de lucidité}

$\rightarrow$ Omar Robert Hamilton

Omar Robert Hamilton est un cinéaste, écrivain et organisateur culturel dans le domaine des documentaires et des fictions. Il a contribué à la création du collectif Mosireen du Caire et travaille sur la documentation, l'archivage et l'enregistrement visuel de la révolution égyptienne sous différentes formes. Il est également à l'origine du festival annuel palestinien de littérature, qui cherche à contrer les différentes politiques d'apartheid d'Isrä̈l et le discours international qui les entoure. Son dernier court-métrage, Though I Know the River is Dry (2013), a rempotté le pix UIP (Roterd), le prix du (2013), a renportéle prix UIP (Rottew the Riveris Dry (2013), a remertéle pix monde arabe (Abu Dhabi) et le prix du meilleur courtmétrage (Yerevan). Omar Robert Hamilton ecrit semirégulièrement pour le collectif de journalistes égyptien Mada Masr et occasionnellement pour le blog Londo Review of Books. Son premier roman, The City Always Wins, sur ces instants de la révolution que l'on ne peut capture dans une archive, sera publié en 2017. L'essai « Moments of clarity" est apparu dans le volume Translating Dissent (Baker 2016) etest republié iciavecl'autorisation Del'auteur et de l'éditeur. www.orhamilton.com

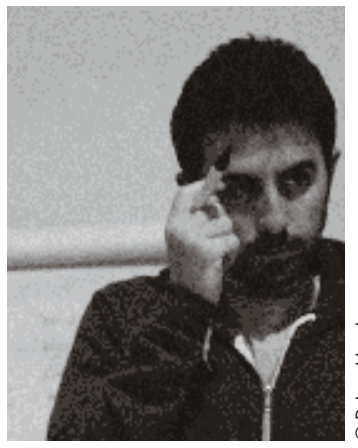

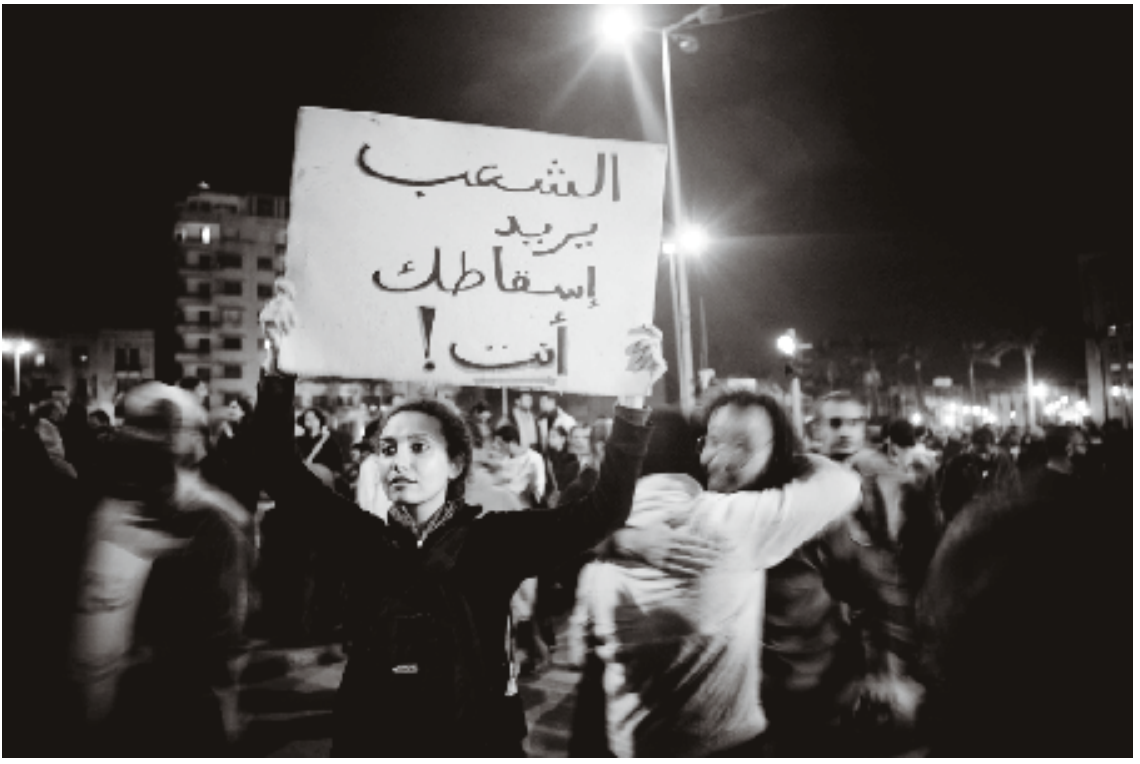

- Manifestations place

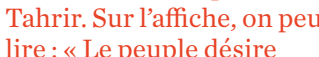
VOTRE chute.»

l est difficile d'être honnête lorsque l'on parle d'un déchirement. Difficile d'être juste. Si vous m'aviez demandé en 2012 sije pensais que la traduction était importante,j'aurais répondu avec enthousiasme qu'elle l'était, que bien sûr elle l'était qu'établir des connexions entre les luttes était essentiel au mouvement global qui prenait forme. J'aurais ajouté que nous avons également la chance, en Egypte, que notre lutte, notre immédiate lutte, donne cette impression qu'elle sera gagnée ou perdue dans l'arène nationale. Que nous ne sommes pas la Palestine et que l'opinion mondiale, au sens le plus large du terme, est utile, mais non-essentielle à nos victoires.

Je comprends encore cette position. J'y pense avec bienveillance. Mais je ne bataille.

La révolution égyptienne de 2011 à 2013 était glorieuse dans sa différence momentanée, dans son caractère inattendu et dans sa lucidité. Ces 18 premiers jours, avec ces claires doléances, le spectacle parlait de lui-même et il n'y avait nul besoin de traduction. Il devait partir. La grande communion de la société égyptienne, des jeunes et des vieux, des laïques et des islamistes (certains), tous unis sous une formule simple : pars. C'était séduisant. Bien sûr que ça l'était. Rien n'est plus simple que de dire Non, que de jeter une pierre sur votre oppresseur. Mais les complexités, les éventuelles fractures devaient se faire jour. Avons-nous choisi de ne pas les voir ? Ou les avons-nous vues et avons-nous pensé qu'elles choisi de écén pouvaient être dépassées? Il est si difficile de se souvenir maintenant, dans ce 
Le spectacle, tel qu'il était, n'avait besoin d'aucune réinterprétation, d'aucun intermédiaire. Il était indéniable.

Nous vivons une époque sans imagination. Dans un moment global sans possibilité. Nous vivons à la fin des temps, au pic, à l'apogée, prêts pour la chute. Nous vivons au bord de l'anéantissement. Nous vivons au-dessus de nos moyens. Nous vivons sans direction. Et c'est ainsi que ces mois de 2011 ont semblé, d'une certaine façon, inévitables, alors que des jeunes se rassemblaient dans le monde, certain luttant pour leur espace public, pour leurs droits, pour leur avenir, du Wisconsin à San'a. La question n'était pas de savoir si un changement était nécessaire ; le changement était arrivé.

Ces aspirants agents du changement pouvaient communiquer les uns avec le autres comme jamais auparavant. Appels Skype, vidéos YouTubes, flux Twitter Indymedias, fils de discussion par e-mail, brochures bilingues, affiches solidaires un nouveau trafic de mots et d'informations est apparu, circulant pour la première fois depuis des siècles du Sud au Nord et du Nord au Sud. Une nouvelle égalité était arrivée entre les jeunes du monde privés de leurs droits.

Mais au fur et à mesure de la progression vacillante dans les mois suivants, dans les divisions du pouvoir et les corruptions des promesses, dans les noudans les divisions du pouvelles constructions des anciennes réalités des militants et des islamistes, le langage a alors réintégré le monde. L'indéniable moment de lucidité ne dure jamais. Des explications étaient demandées, des contextes étaient nécessaires Nos camarades en Espagne et en Grèce étaient dans le flou. Quel était le rôle de l'armée ? Pourquoi a-t-elle été encensée ? Les Frères sont-ils amis ou ennemis de la révolution? Les forces des institutions se réorganisaient. Obama, d'une hypocrisie toujours inégalée, déclare : «Nous devons apprendre à nos enfants a devenir comme les jeunes égyptiens.» Vodafone exhibe fièrement ces mots dans l'aéroport du Caire. La révolution est désormais une marchandise. Elle nous vend du Pepsi, des comptes en banque et les futurs présidents. La langue et l'image de la jeunesse révolutionnaire sont colonisées, transformées en marchandise, de la je, aseptises, ext lamme quelque chose de facilement compréhensible, quelque chose de «bon», quelque chose du passé, quelque chose de terminé. Et lentement, sûrement, ils ont élabor leurs plans contre nous.

Il n'y a jamais eu de révolution totalement réussie, et il n'y en aura jamais.

$* * * *$

Nombre de mes collègues de Mosireen en qui j'ai confiance et que j'admire ont contribué à ce volume. Ils parlent, entre autres, des exigences pratiques du travail effectué, que nous avons réalisé, pour transmettre ce que nous pouvions de la révo- lution à la fois en Égypte et à l'étranger. Mais je ne peux m'empêcher de penser à ce que la révolution n'a pas fait, à ce qui doit se produire la prochaine fois. Nous avons fait confiance à l'inconnu. C'était exaltant, mais c'était facile. Facile de ne pas savoir ce qui allait ensuite se passer, de savoir que cela doit ne pas être ça.

A ce moment dans l'inconnu, il semblait que les images d'Occupy, de $15 M$ et des émeutes de Londres n'avaient besoin d'aucune traduction. Nous étions tous concernés. Mais si nous en avions su plus, si nous avions lu davantage, si nous avions eu la possibilité de parler de planifier et de conspirer, les choses auraient-elles été différentes? Nous avons appis des a cuces les uns des auté différentes? Nous avons appris des astuces les uns des autres, echangé des plaisanteries les uns avec les autres, apporté une force morale les uns aux autres. Nous avons contesté ensemble. Mais nous ne savons toujours pas comment prendre le pouvoir. Ni même si nous le voulons.

À Athènes, j'ai appris la signification du mot martyr. uáptuc. Témoin. C'était en septembre 2011, et les visages, sur les affiches, de ceux qui etaient tombés, affiches qui ceignaient la place Tahrir durant ces 18 premiers jours, étaient encore présents dans nos mémoires. Martyr. Témoin. Comme en arabe. Les racines de chahid se trouvent dans son témoignage. Son observation, sa présence et sa parole. Elle meurtà cause de son témoignage, de ses mots, de son langage de la vérité.

En regardant le visage d'Alexandros, dans son petit mémorial d'Exarcheia, j'ai senti combien ces deux lieux avaient dû être proches autrefois. Athènes et Le Caire L'Égypte et la Grèce. Que de siècles d'échanges, que de connexions et catalyseurs innombrables et inimaginables sont passés entre ces deux lieux pendant des millénaires. La mer n'était pas un obstacle, mais plutôt un maillage évolutif qui liait les Méditerranéens entre eux, une lignée tumultueuse, vivante. Et si vous la traversiez, vous restiez; au moins pour un temps. Combien de temps resteriez-vous aujourd'hui avant le prochain départ d'easyJet? Comment cette Athènes serait-elle traduite pour vous? Ses récits et ses traumatismes ? Comment se comprendre avec ces appels Skype et vidéos YouTube? Le méditerranéen a été remplacé par la langue anglaise comme interface commune, notre principal, et flexible, lien. L'arabe et le grec ne comme ince grec ne se rencontrent plus que rarement. Nous affirmons que nos luttes sont connectées, que nous sommes connectés, mais ces téléphones etces avions ne nous rendent-ils pas plus divisés que jamais?

Justice. Revanche. Punition. Châtiment. Vengeance. Vendetta. Dette de sang. Lequel de ces mots peut emplir la gorge de la mère d'un martyr autant que son appel de lamentation pour قصاص (qasas) lorsque tout le reste a été perdu ? Lequel vous fera pleurer avec elle lorsque la procession funéraire quittera le cimetière?

Je n'ai pas pleuré pour Alexandros.

La contestation est-elle suffisante ? Être en opposition est le destin du contesus ne nous accrocherions pas, ou th $j e$ ne m'accrocherais pas, au pouvoir si nous le prenions. Pourtant, nous affirmons que nous ne pouvons pas demander des libertés à ceux sur le trône, que nous devons 
nous en emparer. Ce paradoxe est central. Mais sinous devons parler, sinous devons

contester, alors oui, nous devons nous traduire pour mieux entendre ce que l'autre a à dire. Nous devons contrer la nature irréfléchie de notre époque. Nous ne gagnerons jamais complètement. Cela ne sera jamais fini. Et nous ne pouvons pas simplement continuer à dire Non. S'il doit y avoir un discours, il doit insuffler quelque chose de nouveau, quelque chose qui n'a toujours pas de langage pour la réalité qu'il peut créer. I

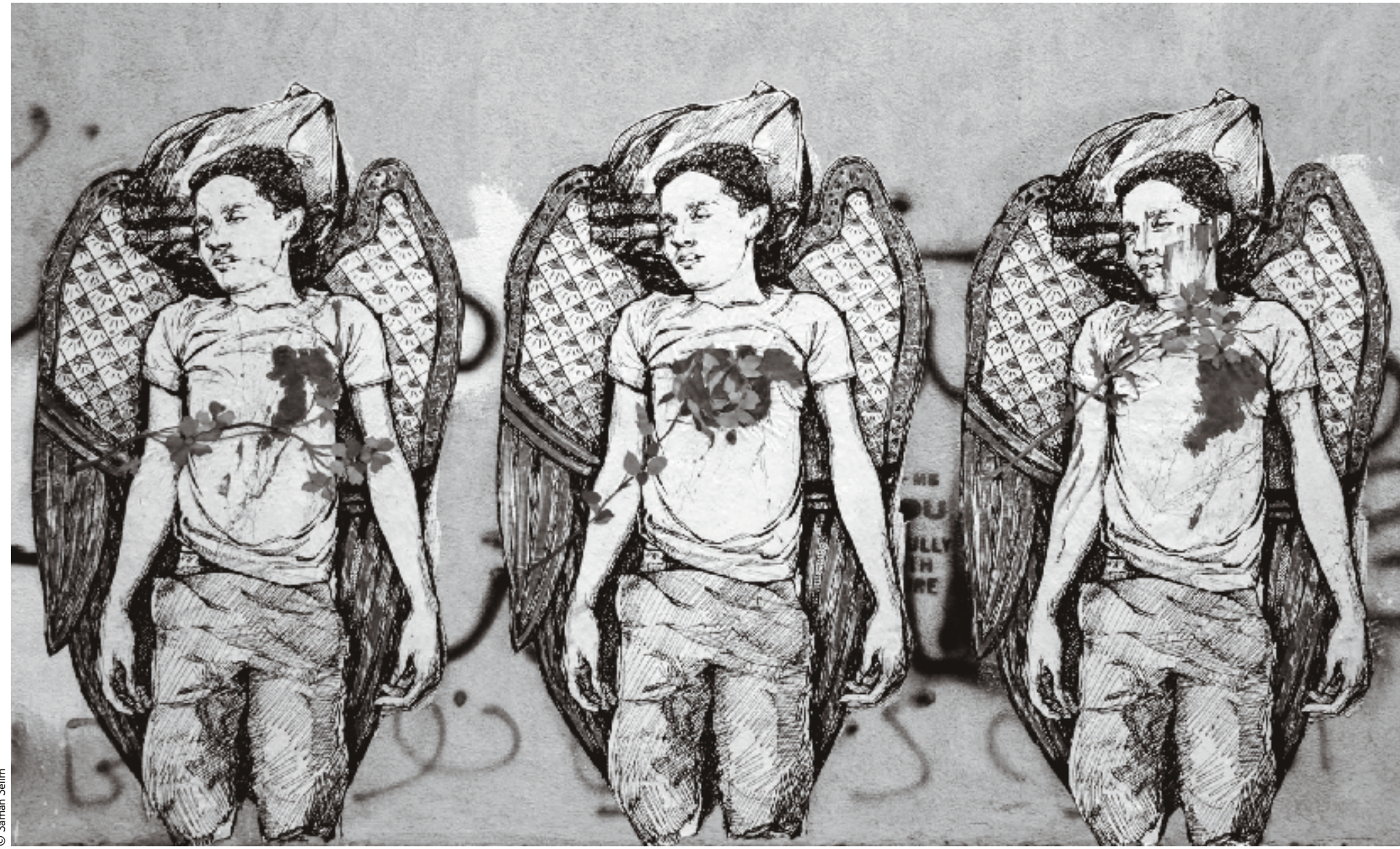

